\title{
Understanding our Galaxy: from the center to outskirts
}

\author{
Z. Q. Shen ${ }^{1}$, Y. Xu ${ }^{2}$, J. L. Han $^{3}$ and X. W. Zheng ${ }^{4}$ \\ ${ }^{1}$ Shanghai Astronomical Observatory, Shanghai 200030, China \\ email: zshen@shao.ac.cn \\ ${ }^{2}$ Purple Mountain Observatory, Nanjing 210008, China \\ email: xuye@mpifr-bonn.mpg.de \\ ${ }^{3}$ National Astronomical Observatories, Beijing 100012, China \\ email: hjl@bao.ac.cn \\ ${ }^{4}$ Department of Astronomy, Nanjing University, Nanjing 210093, China \\ email: xwzheng@nju.edu.cn
}

\begin{abstract}
We describe the efforts to understand our Milky Way Galaxy, from its center to outskirts, including (1) the measurements of the intrinsic size of the galactic center compact radio source Sgr A*; (2) the determination of the distance from the Sun to the Perseus spiral arm; and (3) the revealing of large scale global magnetic fields of the Galaxy.

With high-resolution millimeter-VLBI observations, Shen et al. (2005) have measured the intrinsic size of the radio-emitting region of the galactic center compact radio source Sgr $\mathrm{A}^{*}$ to be only $1 \mathrm{AU}$ in diameter at $3.5 \mathrm{~mm}$. When combined with the lower limit on the mass of Sgr A*, this provides strong evidence for Sgr A* being a super-massive black hole. Comparison with the intrinsic size detection at $7 \mathrm{~mm}$ indicates a frequency-dependent source size, posing a tight constraint on various theoretical models.

With VLBI phase referencing observations, $\mathrm{Xu}$ et al. (2006) have measured the trigonometric parallax of $\mathrm{W} 3 \mathrm{OH}$ in the Perseus spiral arm with an accuracy of $10 \mu$ as and also its absolute velocity with an accuracy of $1 \mathrm{~km} \mathrm{~s}^{-1}$. This demonstrates the capability of probing the structure and kinematics of the Milky Way by determining distances to $12 \mathrm{GHz}$ methanol $(\mathrm{CH} 3 \mathrm{OH})$ masers in star forming regions of distant spiral arms and Milky Way's outskirts.

With pulsar dispersion measures and rotation measures, Han et al. (2006) can directly measure the magnetic fields in a very large region of the Galactic disk. The results show that the largescale magnetic fields are aligned with the spiral arms but reverse their directions many times from the most inner Norma arm to the outer Perseus arm.
\end{abstract}

Keywords. Galaxy: center, Galaxy: structure, black hole physics, masers, pulsars: general, techniques: high angular resolution, astrometry, stars: distances, stars: kinematics, ISM: magnetic fields, ISM: structure

\section{Introduction}

The rapid development of astronomical instruments and techniques has greatly enlarged our knowledge of the structure of Milky Way galaxy, from its center to outskirts. Here, we report some progress made over the past decade, including the measurement of the intrinsic size of the compact radio source $\mathrm{Sgr} \mathrm{A*}$ with the millimeter-VLBI observations, the high-accuracy distance measurement to a massive star forming region $\mathrm{W} 3 \mathrm{OH}$ in the Perseus spiral arm using the trigonometric parallax from the phase-referencing VLBI observations, and the direct measurement of magnetic field in a large region of the Galactic disk using the dispersion measures and rotation measures of pulsars in the Galaxy. 


\section{The size of Sgr $A^{*}$ at the Galactic center}

Sgr A*, an extremely compact non-thermal radio source at the Galactic Center, has been widely recognized as the best and closest candidate for a super massive black hole (SMBH) ever since its first identification in 1974 (Balick \& Brown 1974). The accurate determination of its mass and size is of great importance in testing the SMBH hypothesis. The precise determination of the orbital motions of about a dozen stars at the immediate neighborhood of Sgr A* has provided compelling evidence for the existence of a dark mass of $4 \times 10^{6}$ solar masses within a radius of $45 \mathrm{AU}$ (Ghez et al. 2005; Schödel et al. 2002).

However, past attempts to measure Sgr A* structure with VLBI observations suffered, at long centimeter wavelengths, from the angular broadening caused by the diffractive scattering by the turbulent ionized interstellar medium and, at short millimeter wavelengths, from the large uncertainty in the data calibration due to the low elevation angles at which $\mathrm{Sgr} \mathrm{A}^{*}$ is visible at the telescopes in the northern hemisphere. To improve the accuracy of the observed source structure measurements, we developed a model fitting method by implicitly using the amplitude closure relation (Shen et al. 2003). We then applied this model fitting procedure to various VLBA observations of Sgr A* made at wavelengths from $6 \mathrm{~cm}$ to $3.5 \mathrm{~mm}$ over the time range from 1994 to 2004 .

By fitting the apparent sizes measured at five wavelengths quasi-simultaneously, we were able to revise the 2-dimensional scattering structure (Shen et al. 2005). Then, by subtracting in quadrature the extrapolated scattering size, we successfully detected the intrinsic size of Sgr $\mathrm{A}^{*}$ at $3.5 \mathrm{~mm}$ to be $\approx 1 \mathrm{AU}$ in diameter. Together with a lower limit on the mass of Sgr $A^{*}$ of $0.4 \times 10^{6}$ solar masses from the study of the proper motion of Sgr A* itself (Reid \& Brunthaler 2004), a size of 1 AU sets an extraordinarily high mass density for Sgr A*, strongly arguing for the SMBH nature of Sgr A* (Shen et al. 2005).

Intriguingly, a size of $1 \mathrm{AU}$ for $\mathrm{Sgr} \mathrm{A}^{*}$ is about twice the diameter of the shadow caused by the strong gravitational bending of light at the very vicinity of a 4 million solar masses SMBH. Simulation of the black hole shadow image and the corresponding visibility analysis indicate that future sub-millimeter VLBI experiments are critical and promising to probe the Galactic center black hole (Huang et al. 2007). We are on the verge of resolving the Galactic Center SMBH.

\section{The distance to W3OH in the Perseus spiral arm}

Revealing the true plan-view of the Milky Way has been a challenge for nearly a century. We know less about the structure of our own galaxy than many nearby galaxies like Andromeda galaxy simply because we are embedded inside our galaxy and interstellar dust partially blocks our view.

Observations toward HI clouds and HII regions as well as millimeter-wave observations of molecules (e.g. CO) have revealed coherent, large-scale structures, which are probably spiral arms in the Milky Way. Outlines of spiral arms were then drawn by the locations of HI clouds, HII regions and molecular clouds with their kinematic distances. These distances are based on a model of the rotation of the Milky Way (Georgelin \& Georgelin 1976). However, such a model is affected by some factors, such as the existence of a non-circular rotation and the streaming motions of objects. This can result in a large uncertainty of kinematic distance estimates.

To improve the distance determination, we adopted a most direct and reliable method for measuring astronomical distance known as trigonometric parallax measurement. In practice, we have performed the phase-referencing VLBI observations of the $12 \mathrm{GHz}$ methanol masers in the massive star-forming region $\mathrm{W} 3 \mathrm{OH}$ in the Perseus arm, the nearest arm outward from the Sun. We observed strong and compact maser spots as the 
reference sources and switched rapidly between masers and three distant quasars. The atmospheric and ionospheric delay errors in the VLBA correlator (about $10 \mathrm{~cm}$ in the zenith) were measured during the observations and corrected in the post data processing. Relative position uncertainties were typically about $50 \mu$ as for the quasars and $10 \mu$ as for the masers. Parallax curves of 9 masers relative to 3 quasars were obtained and fitted by the five required parameters, one for the parallax and two parameters for the proper motion in each of the coordinates. This gives a parallax of $\mathrm{W} 3 \mathrm{OH}$ of $0.512 \pm 0.010$ mas (Xu et al. 2006). The corresponding distance is $1.95 \pm 0.04 \mathrm{kpc}$. The distance uncertainty is only $2 \%$, a factor of 100 better than by the Hipparcos satellite. The Perseus arm has a kinematic distance of about $4 \mathrm{kpc}$ and a luminosity distance of $2.2 \mathrm{kpc}$ (Humphreys 1978). Our parallax measurement of $\mathrm{W} 3 \mathrm{OH}$ has now resolved this long-standing discrepancy.

Moreover, the demonstration observations of $\mathrm{W} 3 \mathrm{OH}$ proved the possibility of reconstructing of our own Galaxy within the size of about $20 \mathrm{kpc}$. Currently, a large project of measuring parallaxes and proper motions of both $12 \mathrm{GHz}$ methanol and $22 \mathrm{GHz}$ water maser sources in other regions of the Galaxy is ongoing (Reid, this proceedings). This will enable the mapping of the spiral structure with a great accuracy and further help to determine the distribution of dark matter in the Milky Way.

\section{The Galactic magnetic fields on large scales}

Magnetic fields in a large part of the Galactic disk have been delineated by Faraday rotation data of pulsars, which give a measure of the line-of-sight component of the magnetic field. Pulsars have the advantage of being spread throughout the Galaxy at known distances, allowing a direct mapping of 3-dimensional magnetic field.

For a pulsar at a distance $D$ (in pc), the rotation measure (RM in radians $\mathrm{m}^{-2}$ ) is given by $\mathrm{RM}=0.810 \int_{0}^{D} n_{e} \mathbf{B} \cdot d \mathbf{l}$, where $n_{e}$ is the electron density in $\mathrm{cm}^{-3}, \mathbf{B}$ is the vector magnetic field in $\mu \mathrm{G}$ and $d \mathbf{l}$ is an elemental vector along the line of sight (positive RMs correspond to fields directed toward us) in pc; and the dispersion measure is $\mathrm{DM}=\int_{0}^{D} n_{e} d l$. Thus, pulsars give a direct estimate of the strength of the field through normalization by DM. Furthermore, we can directly estimate the field strength in a given region along the similar lines of sight from the gradient of RM and DM, i.e.

$$
\left\langle B_{\|}\right\rangle_{d 1-d 0}=1.232 \frac{\Delta \mathrm{RM}}{\Delta \mathrm{DM}}
$$

where $\left\langle B_{||}\right\rangle_{d 1-d 0}$ is the mean line-of-sight field component in $\mu \mathrm{G}$ for the region between distances $d 0$ and $d 1, \Delta \mathrm{RM}=\mathrm{RM}_{d 1}-\mathrm{RM}_{d 0}$ and $\Delta \mathrm{DM}=\mathrm{DM}_{d 1}-\mathrm{DM}_{d 0}$.

Up to now, RMs of 550 pulsars have been observed (e.g., Han et al. 1999, Han et al. 2006). Most of the new measurements lie in the fourth and first Galactic quadrants and are relatively distant, enabling us to investigate the structure of the Galactic magnetic field over a much larger region than it was previously possible. We detected counterclockwise magnetic fields in the inner Norma arm (Han et al. 2002). A more complete analysis for the fields near the tangential regions of most probable spiral of our Galaxy (Han et al. 2006) gives such a picture for the coherent large-scale fields aligned with the spiral arm structure in the Galactic disk: magnetic fields in all inner spiral arms are counterclockwise when viewed from the North Galactic pole. On the other hand, at least in the local region and in the inner Galaxy of the fourth quadrant, there is good evidence that the fields in inter-arm regions are coherent, but clockwise in orientation. Thus, there are at least two or three reversals in the inner Galaxy, probably occurring near the boundary of the spiral arms. The magnetic field in the Perseus arm can not be well determined. The negative RMs for distant pulsars and extra-galactic sources in fact suggest that the 
inter-arm fields both between the Sagittarius and Perseus arms and beyond the Perseus arm are predominantly clockwise.

With much more pulsar RM data available now, Han et al. (2006) were able to measure, rather than model, the regular field strength near the tangential regions in the 1st and 4th Galactic quadrants, and then plot the dependence of regular field strength on the Galactic-radii. Although the "uncertainties", which in fact reflect the random fields, are large, the tendency is clear that fields get stronger at smaller Galactocentric radii and weaker in inter-arm regions. To parameterize the radial variation, an exponential function was used as follows, which not only gives the smallest $\chi^{2}$ value but also avoids a singularity at $R=0$ (for $1 / R$ ) and non-physical values at large $\mathrm{R}$ (for the linear gradient). That is, $B_{\mathrm{reg}}(R)=B_{0} \exp \left[\frac{-\left(R-R_{\odot}\right)}{R_{\mathrm{B}}}\right]$, with the strength of the large-scale or regular field at the Sun, $B_{0}=2.1 \pm 0.3 \mu \mathrm{G}$ and the scale radius $R_{\mathrm{B}}=8.5 \pm 4.7 \mathrm{kpc}$.

From the RM distribution in the sky, Han et al. $(1997,1999)$ identified the striking antisymmetry in the inner Galaxy with respect to the Galactic coordinates after removing the RM "outliers" compared to their neighborhoods. Such an antisymmetry should be a result from the azimuthal magnetic fields in the Galactic halo with reversed field directions below and above the Galactic plane. Such a field can be produced by an A0 mode of dynamo. The observed filaments near the Galactic center should result from the dipole field in this dynamo scenario. The local vertical field component of $0.2 \mu \mathrm{G}$ (Han \& Qiao 1994, Han et al. 1999) may be part of this dipole field in the solar vicinity. At present, we have observed another 1700 radio sources in the Northern sky by the Effelsberg 100 m telescope (Han, Reich et al. 2007, in preparation), and we wish to do more in the Southern sky at Parkes, so that the RM sky can be described quantitatively.

\section{Acknowledgements}

Chinese astronomers have dedicated to the reported study of the structure of our Galaxy using numerous radio telescopes over the globe. Many results would not be possible without kind help from our international collaborators including K. Y. Lo, R. N. Manchester, M. Reid. We appreciate very much for their long term support and cooperation. Research of JLH is supported by NNSFC under grant numbers 10473015, 10521001 and 10773016. YX is supported by NNSFC under grant numbers 10673024 and 10621303 , and NBRPC (973 Program) under grant 2007CB815403. ZQS is supported in part by grants 10573029, 10625314, 10633010, and 06XD14024. ZQS acknowledges the support by the One-Hundred-Talent Program of the Chinese Academy of Sciences.

\section{References}

Balick, B. \& Brown, R. L. 1974, ApJ, 194, 265

Georgelin, Y. M. \& Georgelin, Y. P. 1976, A\& A, 49, 57

Ghez, A. M. et al. 2005, ApJ, 620, 744

Han, J. L. \& Qiao, G. J. 1994, A\& A, 288, 759

Han, J. L., Manchester, R. N., Berkhuijsen, E. M., \& Beck, R. 1997, A\&̊A, 322, 98

Han, J. L., Manchester, R. N., \& Qiao, G. J. 1999, MNRAS, 306, 371

Han, J. L., Manchester, R. N., Lyne, A. G., \& Qiao, G. J. 2002, ApJ, 570, L17

Han, J. L., Manchester, R. N., Lyne, A. G., Qiao, G. J., \& van Straten, W. 2006, ApJ, 642, 868.

Huang, L., Cai, M., Shen, Z.-Q., \& Yuan, F. 2007, MNRAS, 379, 833

Humphreys, R. M. 1978, ApJS, 38, 309

Reid, M. J. \& Brunthaler, A. 2004, ApJ, 616, 872

Schödel, R. et al. 2002, Nature , 419, 694

Shen, Z.-Q., Liang, M. C., Lo, K. Y., \& Miyoshi, M. 2003, Astron. Nachr. 324, S1, 383

Shen, Z.-Q., Lo, K. Y., Liang M.-C., Ho, P. T. P., \& Zhao J.-H. 2005, Nature , 438, 62

Xu, Y., Reid, M. J., Zheng, X. W., \& Menten, K. M. 2006, Science, 311, 54 